\title{
Epidermal Smad4 Deletion Results in Aberrant Wound Healing
}

\author{
Philip Owens, ${ }^{*}$ Erin Engelking, ${ }^{*}$ Gangwen Han, ${ }^{* \dagger}$ \\ Sarah M. Haeger, ${ }^{\dagger}$ and Xiao-Jing Wang ${ }^{\star \dagger}$ \\ From the Departments of Otolaryngology, Dermatology, Cell and \\ Developmental Biology,* Oregon Health Sciences University, \\ Portland, Oregon; and the Department of Pathology, University \\ of Colorado Denver Health Sciences Center, Aurora, Colorado
}

In the present study, we assessed the role of Smad4, a component of the transforming growth factor- $\beta$ signaling pathway, in cutaneous wound repair. Interestingly, when Smad4 was deleted in the epidermis, several defects in wound healing were observed in non-keratinocyte compartments. In comparison with wounded wild-type mouse skin, Smad4-deficient wounds had delayed wound closure and remodeling. Increased angiogenesis and inflammation were found in Smad4-deficient skin; these effects were exacerbated throughout the entire wound healing process. In addition, increased numbers of myofibroblasts but reduced collagen levels were found in Smad4-deficient wounds in comparison with wild-type wounds. Since Smad4 is not a secreted protein, we assessed if the above non-cell autonomous alterations were the result of molecular alterations in Smad4-deficient keratinocytes, which exert paracrine effects on wound stroma. Smad4-deficient skin and wounds had elevated levels of transforming growth factor- $\beta 1$, which have been shown to induce similar phenotypes, as well as of several transforming growth factor- $\beta 1$ target genes, such as matrix metalloproteinases, vascular endothelial growth factor-A, and chemokine (C-C motif) ligand 5. Furthermore, the above pathological and molecular alterations were exacerbated in skin cancer lesions that spontaneously developed from Smad4-deficient skin. Therefore, loss of Smad4 in the epidermis appears to significantly affect the microenvironment during wound healing and carcinogenesis. (Am J Pathol 2010, 176:122-133; DOI: 10.2353/ajpath.2010.090081)

Cutaneous wound healing is a complex yet highly coordinated series of events including the distinct yet overlapping processes of inflammation, re-epithelialization and stromal remodeling (for review see Singer and Clark, $1999^{1}$ ). Inflammation occurs immediately on wounding with the presence of infiltrating neutrophils and macrophages, which will phagocytose debris in the wound and subsequently undergo programmed cell death. Concurrently, keratinocytes from the epidermis begin to reepithelialize into the wound area and form an epidermal "migrating tongue." Once the wound is closed and inflammation subsides, the dermis remodels the granulation tissue (consisting of new connective tissue and tiny blood vessels formed in the wound) and reorganizes the extracellular matrix (ECM). New blood vessels that helped deliver inflammatory leukocytes and nutrients regress and become quiescent. Myofibroblasts, which were activated to secrete the new ECM and growth factors, undergo programmed cell death after synthesizing the newly arrayed collagen. Finally, scar tissue is either resolved or permanently remains at the wound site.

Transforming growth factor (TGF) $\beta$ signaling is one of the major signal transduction pathways regulating all phases of cutaneous wound healing in many cell types. ${ }^{2,3}$ This signaling pathway is largely mediated by Smads (for a detailed review of Smads see Massague, 20054). Smad2 and Smad3 are phosphorylated by TGF $\beta$ receptors and associate with the common partner Smad4, which transmits the TGF $\beta$ signal to the nucleus to regulate TGF $\beta$ transcriptional targets. Many earlier studies suggested that TGF $\beta 1$ is necessary for cutaneous wound healing via the initiation of inflammation, promoting epithelial cell migration, stimulating dermal fibroblast proliferation, and stimulating collagen synthesis and ECM remodeling. 5,6 However, recent in vivo studies have demonstrated the negative effects of TGF $\beta$ signaling on wound healing. ${ }^{6}$

Supported by NIH grants to X.-J.W. P.O. is a recipient of an NIH training grant and supported by the Tartar Medical Trust. E.E. is a Murdock Fellow.

Accepted for publication September 22, 2009.

Supplemental material for this article can be found on http://ajp. amjpathol.org.

Current address for P.O.: Department of Cancer Biology, Vanderbilt University Medical Center, Nashville, TN.

Address reprint requests to Xiao-Jing Wang, M.D., Ph.D., Department of Pathology, University of Colorado Denver Health Sciences Center, Bldg. RC1-N, Rm P28-5128, Mail Box 8104, 12800 E.19th Ave, Aurora, CO 80045. E-mail: XJ.Wang@UCDenver.edu. 
TGF $\beta 1$ has been shown to exacerbate inflammation during wound healing when overexpressed. ${ }^{7}$ Transgenic mice expressing the TGF $\beta 1$ transgene in keratinocytes show delayed healing after burn injury or incisional wounding. ${ }^{6,8,9}$ Consistently, Smad3 knockout mice exhibit accelerated cutaneous wound healing characterized by increased keratinocyte proliferation and migration, and reduced monocyte infiltration. ${ }^{10}$ Conversely, Smad2 overexpression in basal keratinocytes results in abnormalities in epidermal re-epithelialization and wound closure. ${ }^{11}$

The role of Smad4 in wound healing has only been studied in vitro; Smad4-deficient epithelial cells have delayed cell migration, especially in response to the TGF $\beta$ ligand. ${ }^{12}$ In the present study, we examined its in vivo effects on cutaneous wound healing. Our results showed that disruption of Smad4 in the epidermis mainly results in abnormalities in non-keratinocytes during cutaneous wound healing, which were exacerbated in Smad4-deficient skin tumors. Since Smad4 is often lost in skin cancer, ${ }^{13}$ our findings suggest a significant impact of epithelial Smad4 loss on the surrounding microenvironment under pathological conditions.

\section{Materials and Methods}

\section{Animals, Wounding and Wound Morphology, and Spontaneous Skin Squamous Cell Carcinoma Formation}

Smad4 conditional knockout mice were generated as previously described ${ }^{2}$ by crossing Smad4 floxed (fl/fl) mice $^{14}$ with MMTV.Cre ${ }^{15}$ mice. Smad4fl/fl mice without Cre were used as wild-type controls. Smad4 knockout $(K O)$ mice were homozygous for the Smad4 floxed allele and carried one allele of the MMTV.Cre transgene. We have previously shown that in this strain Smad4 deletion in the skin is restricted to keratinocytes. ${ }^{2}$ To further confirm Smad4 deletion is restricted to keratinocytes in the skin, we cross-bred MMTV.Cre/Smad4fl/fl mice with Rosa26 reporter mice ${ }^{16}$ in which the floxed stop sequence is inserted upstream of the $L a c Z$ reporter gene (The Jackson Laboratory, Bar Harbor, Maine) and gene deletion is visualized by $\beta$-gal staining of the skin sections from MMTV.Cre/Smad4fl/Rosa26 mice (Supplemental Figure 1, see http://ajp.amjpathol.org). Twelve-week-old animals were anesthetized and wounded with a full-thickness 6-mm punch biopsy at four positions on the dorsal skin of their flanks. Images of wounds were taken with four surrounding reference objects of known size to calibrate wound area. Images were quantified with ImageJ (National Institutes of Health). Over 40 wounds were analyzed for each time point and respective genotype. Percentage of wound closure was analyzed using a box and whisker plot. Spontaneous skin tumors formed in these mice from 3 to 13 months of age as previously described. ${ }^{2}$ Mice were maintained and bred under pathogen-free barrier conditions. Additionally, animals were observed for up to 10 weeks post-wound healing to monitor for signs of infection. Only non-tumor bearing mice were used for wound healing studies and all appropriate animal care guidelines of the Institutional Animal Care and Use Committee at Oregon Health \& Science University and at University of Colorado Denver were strictly followed.

\section{Antibodies}

The following antibodies were used for immunofluorescence or immunohistochemistry: guinea pig anti-K14 (RDIFitzgerald, Acton, MA, 1:500); Rat anti-F4/80 (Invitrogen, Carlsbad, CA, 1:200); mouse anti- $\alpha$ smooth muscle actin (SMA; Sigma-Aldrich, St. Louis, MO, 1:500); Rat anti-CD31 (BD Pharmingen, San Jose, CA, 1:200); goat anti-proliferating cell nuclear antigen (Santa Cruz Biotechnology, Santa Cruz, CA, 1:100), chicken anti-TGF $\beta 1$ (R\&D, Minneapolis, MN, 1:100); and rabbit anti-mouse/human pSmad2 (Cell Signaling Technology, Danvers, MO, 1:50).

\section{Sample Preparation, Histology, Immunofluorescence, and Immunohistochemistry}

Each animal was given four dorsal flank wounds (anterior left, anterior right, posterior left, posterior right) and every location was used for each type of tissue preparation. Wounds were collected using an 8-mm punch biopsy tool after euthanasia. The first two wounds from euthanized animals were immediately frozen in liquid nitrogen and used for RNA or protein extraction. The third wound was placed in optimal cutting temperature compound and immediately frozen. The fourth wound was fixed in 10\% neutral buffered formalin overnight, changed to $70 \%$ ethanol, embedded into paraffin, and sectioned into 5 $\mu \mathrm{mol} / \mathrm{L}$ sections for staining. Frozen sections were cut at $8 \mu \mathrm{m}$. Frozen or paraffin-embedded wounds were bisected equilaterally before sectioning/embedding. Sections were H\&E-stained (Gil) per standard protocol. Other sections were stained with a Masson trichrome staining kit (Sigma) according to manufacturer instructions. Frozen sections were fixed in $75 \%$ isopropanol for 5 minutes, washed in PBS, and placed in blocking buffer. Paraffin slides were de-waxed in xylene twice for 5 minutes and placed in an ethanol rehydration series including 3-minute washes, which culminated in a PBS rinse. Slides were microwaved in $\mathrm{pH} 6.0$ citrate buffer for antigen retrieval and blocked in 5\% serum of the secondary antibody host. MOM (mouse-on-mouse) blocking agent (Vector Labs, Burlingame, CA) was used as a blocking agent if antimouse IgG was used as a secondary antibody. Sections were incubated with primary antibodies at the dilutions listed above overnight at $4^{\circ} \mathrm{C}$. For immunofluorescence, secondary AlexaFluor antibodies (Invitrogen) were used at a dilution of 1:100 for a 10-minute incubation at room temperature. Slides were washed four times in PBS and mounted in SlowFade containing 4,6-diamidino-2-phenylindole mounting medium (Invitrogen). For immunohistochemistry, the sections were incubated with secondary biotinylated antibodies (4 $\mu \mathrm{g} / \mathrm{ml}$ ) for 30 minutes at room temperature and further detected using Vectastain ABC kit (Vector 
Labs). Hematoxylin QS (Vector Labs) was used as the counterstain.

\section{PicroSirius Red Staining and Imaging}

PicroSirius red staining was performed per previously published protocols with only minor modifications. ${ }^{17}$ Paraffin slides were de-waxed in 100\% xylene and hydrated through a series of ethanol and water washes. The sections were stained in PicroSirius red solution $(0.1 \%$ Sirius red F3B in saturated picric acid solution, Sigma) for 1 hour at room temperature. After 2 quick washes in $0.1 \mathrm{~N}$ acetic acid, the slides were dehydrated using ethanol and xylene washes and mounted with polymount-xylene (Polyscience Inc). Images were taken under a microscope with and without a Polaroid lens.

\section{Quantitative Reverse Transcription-PCR}

Total cellular RNA was isolated from freshly frozen wounds stored at $-80^{\circ} \mathrm{C}$. Wounds were placed in Trizol, minced using scissors and homogenized. RNA was isolated through subsequent chloroform/isopropanol/ethanol purification. Aliquots of RNA were further purified using RNeasy columns (Qiagen, Valencia, CA) coupled with on-column DNA digestion. One hundred nanograms of total RNA was used per well and Brilliant II 1-step QPCR reagent (Stratagene, La Jolla, CA) was used to convert RNA into CDNA and amplified in an M×3000P QPCR machine (Stratagene). VIC-labeled glyceraldehyde-3-phosphate dehydrogenase (Applied Biosystems, Foster City, CA) was used as a normalizing housekeeper gene. 6-Carboxyl-X-Rhodamine (ROX) was used as a reference dye for background fluorescence. The following 5-carboxyfluorescein-labeled Taqman probes (Applied Biosystems) were used: VEGFAMm00437306_m1, CCL5-Mm01302428_m1, CCL20-Mm00444228_m1, TGF $\beta 1$ - Mm03024053_m1, MMP3-Mm00440295_m1, MMP12-Mm00500554_m1, MMP13-Mm00439491_ m1, and MMP14-Mm00485054_m1. Relative quantity or log fold change was calculated using MxPro software V.4 (Stratagene) using all three algorithm enhancements (adaptive baseline, moving average, and amplification-based thresholds).

\section{Keratinocyte Migration Assay}

Low passage primary keratinocytes isolated from newborn mice were plated in six-well culture dishes, grown to confluency, and a standard $200 \mu$ l pipette was used to "wound" the keratinocyte monolayer. Mitomycin-C was added at a concentration of $4 \mu \mathrm{g} / \mathrm{ml}$ for 1 hour to block proliferation and exclude any non-migration contributions such as increased cell number. Images were captured on an inverted microscope with phase contrast.

\section{Statistical Analysis}

Measurements were subjected to a paired two-tailed students $t$-tests. Significance was determined by $P$ values lower than 0.05 .

\section{Results}

\section{Epidermal Specific Smad4 KO Wounds Healed Poorly}

Previously, we mated Smad4 fl/fl mice with MMTV-Cre mice showing that in addition to Smad4 deletion in mammary glands, Smad4 is deleted in hair follicles and the epidermis in the skin. ${ }^{2}$ To further assess the role of Smad4 loss in cutaneous wound healing, we generated four 6-mm fullthickness skin wounds in bigenic MMTV-Cre/Smad4 fl/fl mice (Smad4 KO) and mice lacking MMTV-Cre. When compared with wild-type wounds, Smad4 KO wounds healed poorly (Figure 1A) and were delayed in wound closure (Figure 1, B and C). Three days after the punch biopsy, 53\% of the wound area had healed in wild-type mice (averaged across 68 wounds in 17 mice). In contrast, only $29 \%$ of the wound had healed by three days after the punch biopsy in Smad4 KO mice, averaged across 56 wounds in 14 mice $(P<0.001)$. By day 5 postwounding, wild-type wounds had an average of $70 \%$ of the healed wound area, while Smad4 $K O$ wounds had only healed $52 \%$ of the original wound area $(P<0.001)$.

\section{Smad4-Deficient Keratinocytes Do Not Have Defects in the Length of the Epidermal Migrating Tongue}

Since the gross appearance of wound closure represents wound contraction and/or re-epithelialization, we first assessed if Smad4 KO wounds had a defect in re-epithelialization. Examination of the leading edge of wound epidermis did not reveal a significant difference in the length of the migrating tongue between Smad4 $K O$ and wild-type wounds (Figure 2, A and B). Previous studies have shown that knocking down Smad4 in keratinocytes from both tumors and epidermis causes migration defects in vitro ${ }^{12,18}$. We performed in vitro scratch assays using wild-type and Smad4 KO keratinocytes and observed results similar to previous findings (Figure 2C). Twenty-four and 48 hours after scratching, wild-type keratinocytes showed a migration pattern with a coordinated sheet, whereas Smad4 KO cells migrated individually (Figure 2C). Since Smad4 KO cells are hyperproliferative, ${ }^{12}$ increased proliferation may be the major factor overcoming the migration defect in vivo. Indeed, proliferating cell nuclear antigen staining showed increased proliferative cells in both the migrating epidermis and underlying stroma of Smad4 KO wounds (Figure 2D). These results suggest that in vivo enlarged wound areas of Smad4 KO wounds were not due to delayed epidermal migration, but represent at least in part, poor wound contraction.

\section{Wounds That Lack Epidermal Smad4 Have Enlarged Vessels and Increased Angiogenesis}

Histological examination revealed enlarged blood vessels in Smad4 KO wounds extending dramatically into the 
A
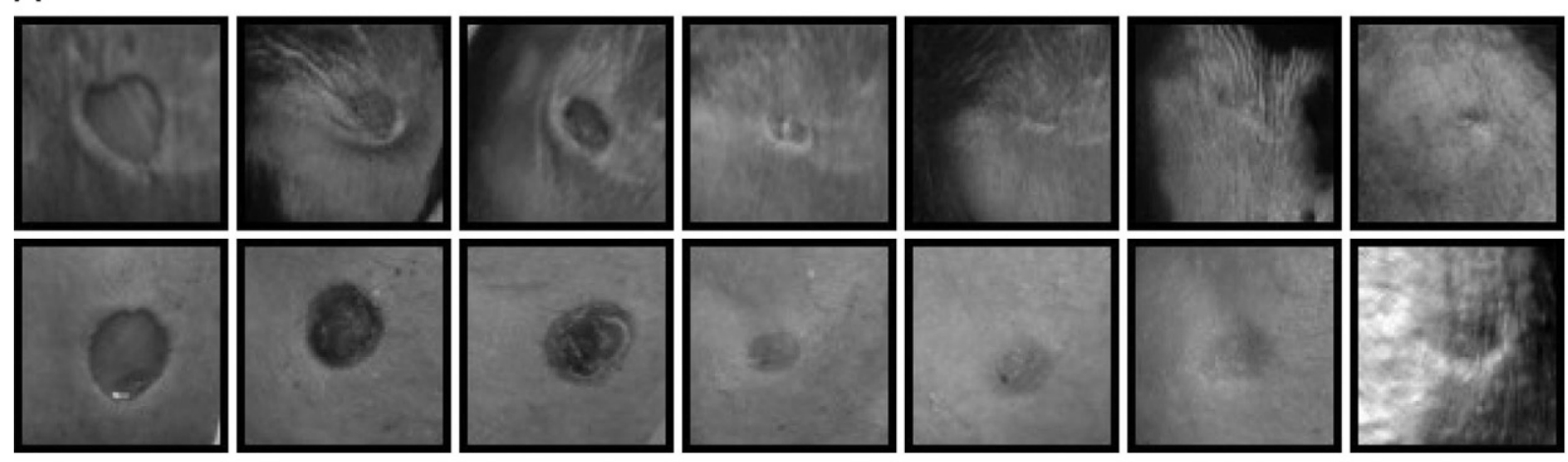

$K O$

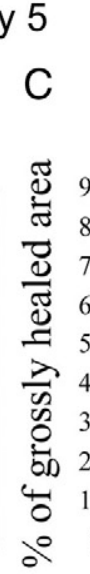

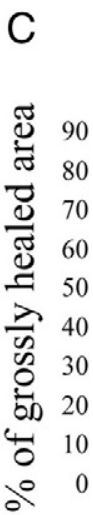

Day 10

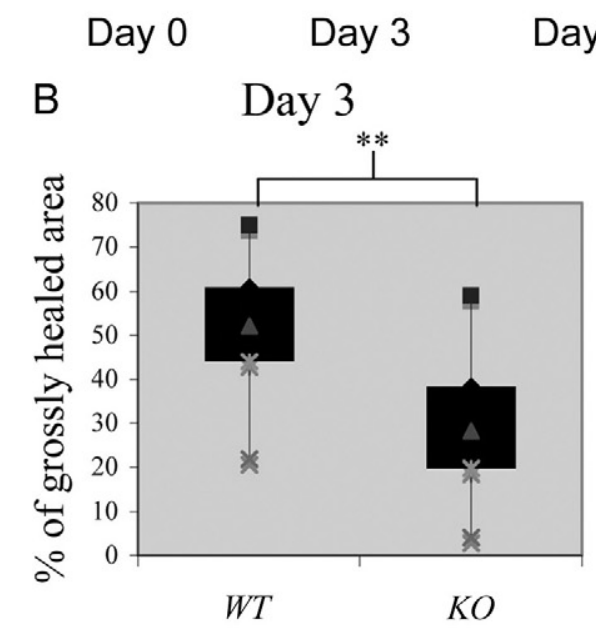

wound bed. These vessels were present throughout the wound healing process, as well as post wounding (Figure $3 \mathrm{~A}$, black arrows). To quantify the blood vessels, we stained for CD31 (PECAM) to mark endothelial cells. Before wounding, more vessels appeared in Smad4 KO skin surrounding collapsed hair follicle epidermoid cysts and throughout the dermis (Figure 3, B and C). Angiogenesis was further increased under the wound margins at day 3 in Smad4 KO wounds (Figure 3, B and C). This is in stark contrast to wild-type wounds, in which the vessels were still restricted to the unwounded area behind the migrating tongue (Figure $3 \mathrm{~B}$, dotted white line). By day 5, both wild-type and Smad4 KO skin exhibited significant angiogenesis behind the migrating tongue (not shown). To further analyze the associated molecular mechanism, we examined VEGFA expression in wound tissues using real-time PCR and found that VEGFA mRNA was significantly increased in Smad4 KO skin before wounding (day 0, Figure 3D). By day 3, when wild-type wounds began to have increased VEGFA, VEGFA mRNA was further increased in Smad4 $K O$ wounds (Figure 3E).

\section{Monocyte/Macrophage Infiltration Increased in Smad4-Deficient Skin and Wounds}

Histology of Smad4 KO wounds revealed an increase in infiltrated leukocytes (not shown). Therefore, we exam- ined markers for inflammatory cells by immunofluorescence in unwounded and wounded wild-type and Smad4 $K O$ skin. We found a dramatic increase in the numbers of F4/80-positive monocyte/macrophages in Smad4 KO skin before wounding (Figure 4A) and throughout the wound healing process, in comparison with wild-type skin and wild-type wounds. Furthermore, chemokines CCL5 and CCL20, which play causal roles in inflammation, ${ }^{19-22}$ were increased 38- and 12-fold, respectively, in Smad4 $\mathrm{KO}$ skin, in comparison with wild-type skin, where both chemokines were scantly detectable (Figure 4, B and C). During wound healing, CCL5 mRNA levels were further increased three- and eightfold in Smad4 KO wounds at day 3 and day 5 , respectively, as compared with wildtype wounds. CCL20 mRNA levels were elevated 30 and 18-fold at day 3 and day 5, respectively, in Smad4 KO wounds, in comparison with wild-type wounds (Figure 4, $\mathrm{D}$ and E). Markers for other inflammatory cells such as neutrophils, dendritic cells, and T-cells did not show a significant difference between Smad4 KO and wild-type wounds (data not shown).

\section{Epidermal Smad4 Deletion Caused Multiple Abnormalities in the Dermis and Persistent Scarring of Wounds}

We observed that $\alpha \mathrm{SMA}+$ myofibroblasts, which are responsible for the generation of new ECM following inflam- 
A Day 3

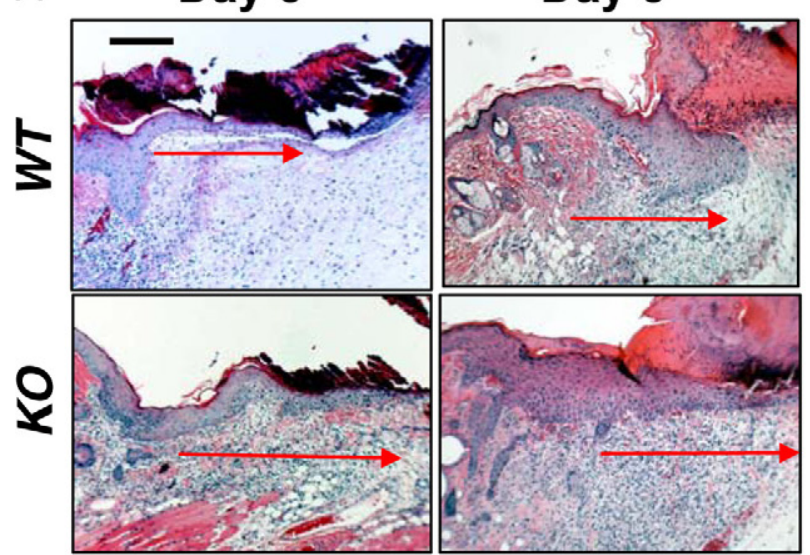

B
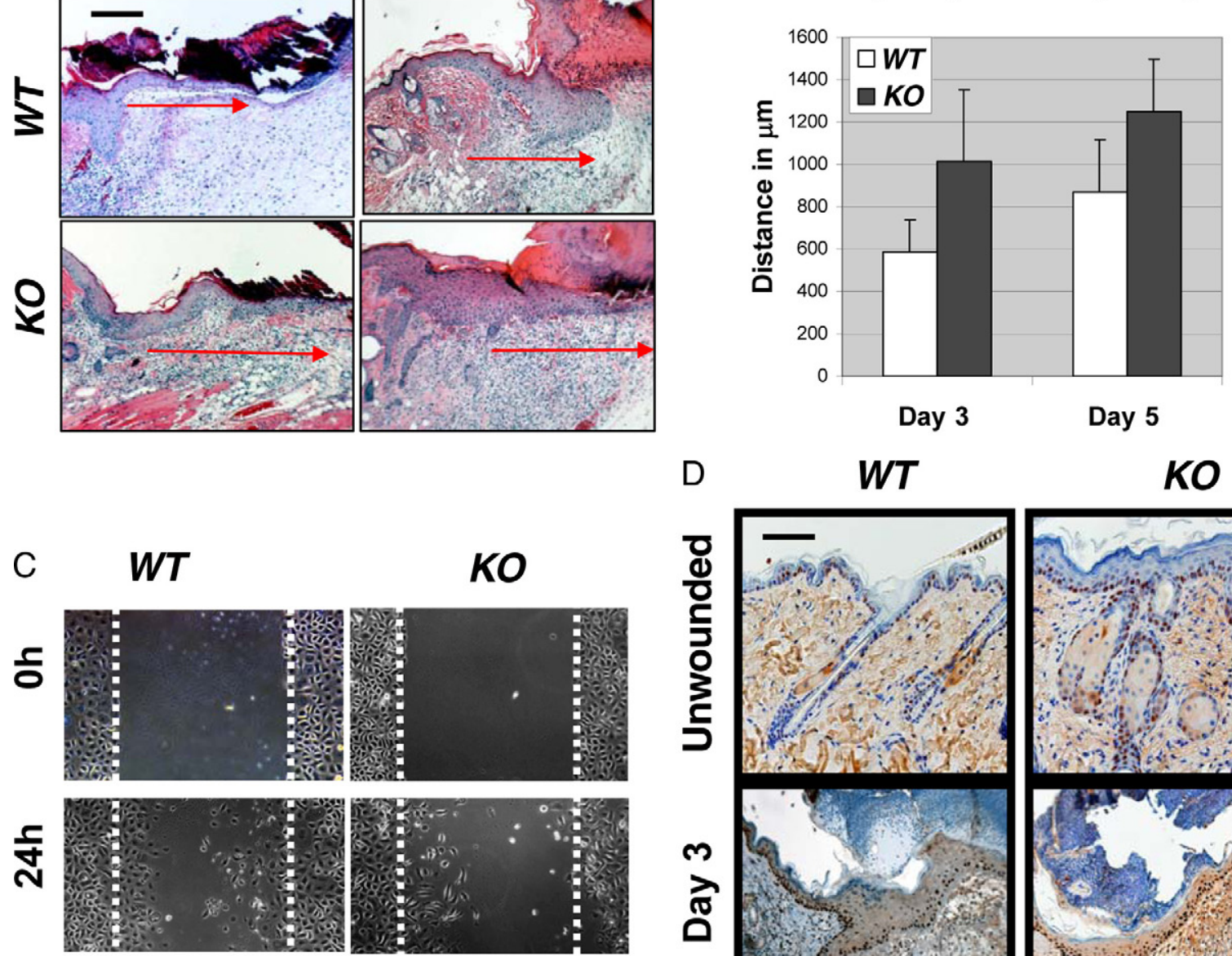

文
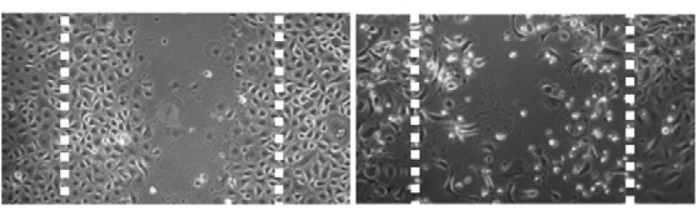

D

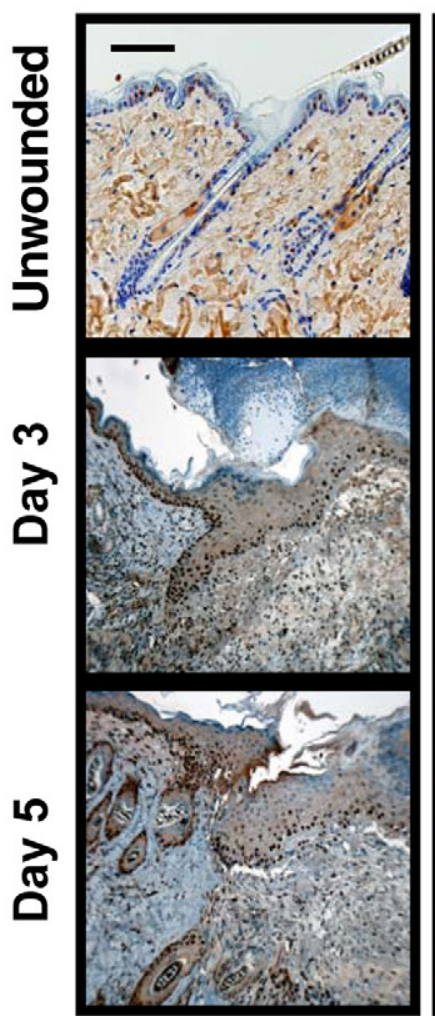

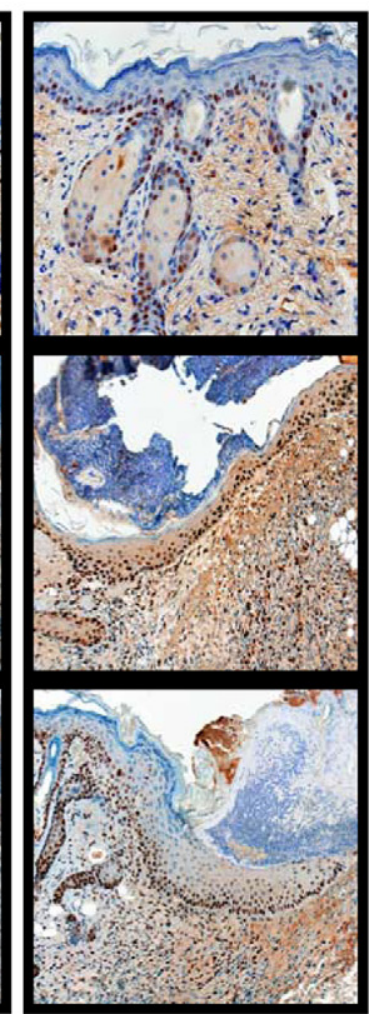

Figure 2. In vitro migration defects of Smad4 deficient keratinocytes can be overcome in vivo. A: H\&E sections of the wounds with migrating tongues, days 3 and 5. The lengths of the migrating tongues are shown with corresponding red arrows. B: Quantification of the migrating tongue lengths. A minimum of five tongues from each genotype were measured for each time point; differences were not statistically significant. C: In vitro migration assay showing Smad4 KO keratinocytes migrated individually, but failed coordinated migration. The dotted lines highlight the scratched margin areas. D: Proliferating cell nuclear antigen staining showing increased proliferation in Smad4 KO epidermis in non-wounded skin, and in Smad4 KO epidermis and stroma in wounded skin compared with wild-type (WT) controls. Scale bars in (A) and (D) represent $200 \mu \mathrm{m}$ for all panels.

mation, were significantly increased in the Smad4 KO stroma adjacent to the wound margin (Figure 5). At day 3 following wounding, Smad4 KO wounds accumulated myofibroblasts while wild-type controls did not (Figure 5A). By day 5 , the numbers of myofibroblasts between wild-type and Smad4 KO wounds were comparable (Figure 5A). However, myofibroblasts were restricted to the underneath area of the migrating tongue in wild-type wounds, but were more spread out in Smad4 KO wounds (Figure 5A). By 14 days after wounding, when $\alpha \mathrm{SMA}+$ cells were restricted to the vessels in wild-type skin, Smad4 KO wounds exhibited many $\alpha \mathrm{SMA}+$ myofibroblasts both in vessel walls and outside of vessels (Figure 5A). Since no differences in the number of apoptotic cells were found between wild-type and Smad4 KO wound stroma (not shown), the accumulation of myofibroblasts could be a consequence of increased proliferation of these cells (Figure 2). Further, Masson trichrome staining revealed that the ECM appeared to be less organized and that granulation tissue appeared to be expanded in Smad4 KO wounds (Figure 5B). Excessive 


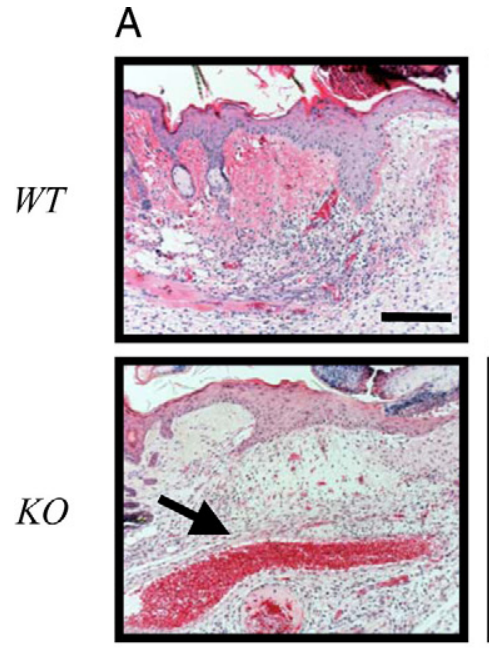

Day 3
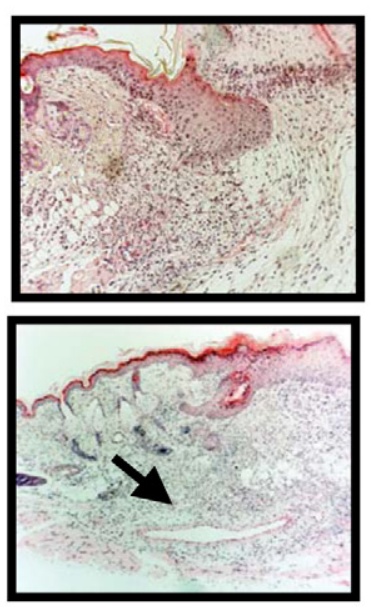

Day 5
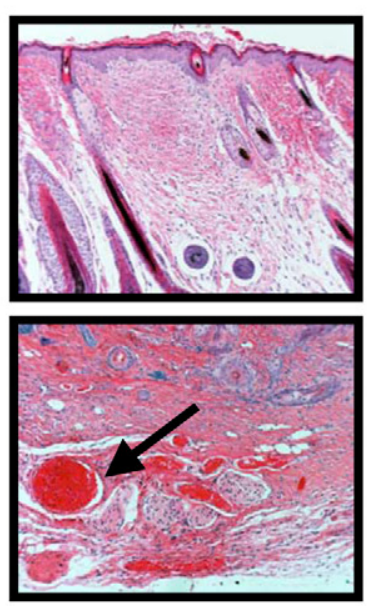

Day 14
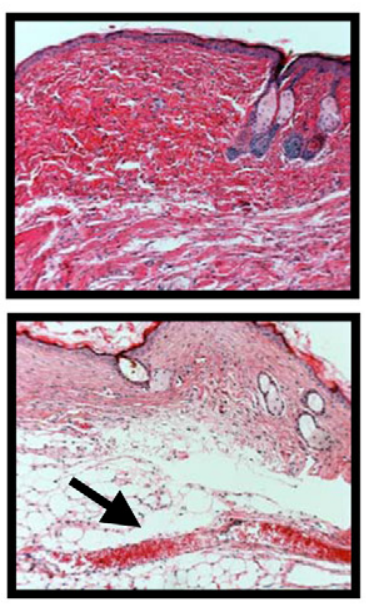

Day 42

B

CD31/K14/DAPI

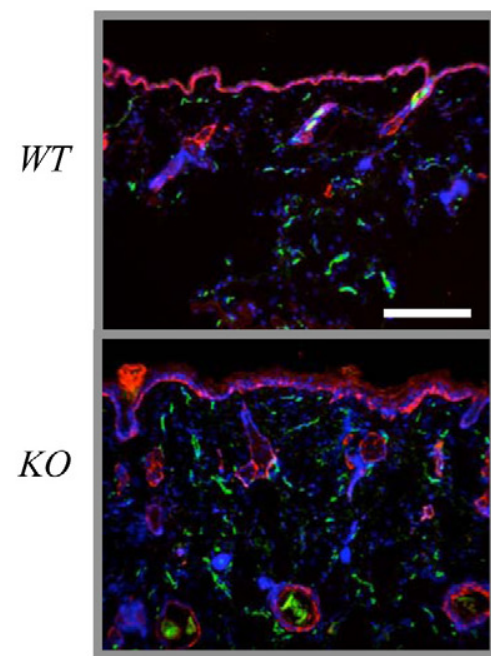

Day 0

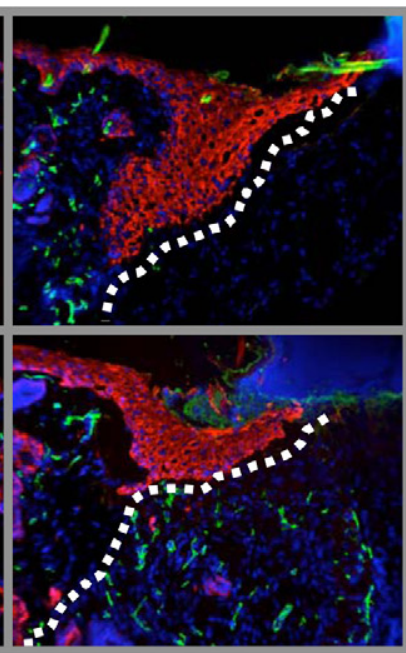

Day 3

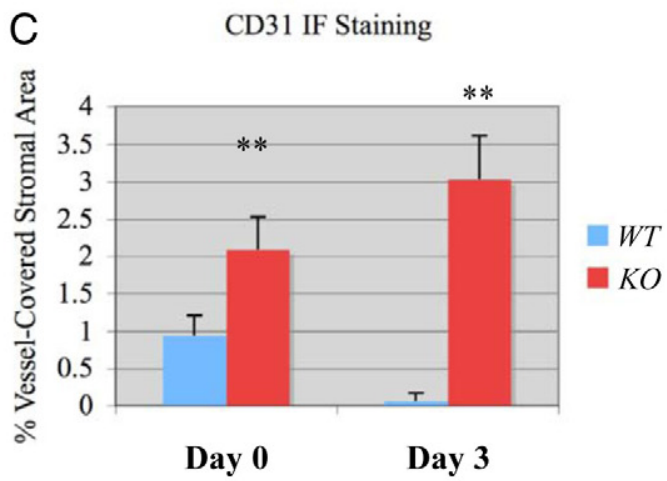

D VEGFA mRNA in Unwounded skin

E VEGFA mRNA in Day 3 Wounds
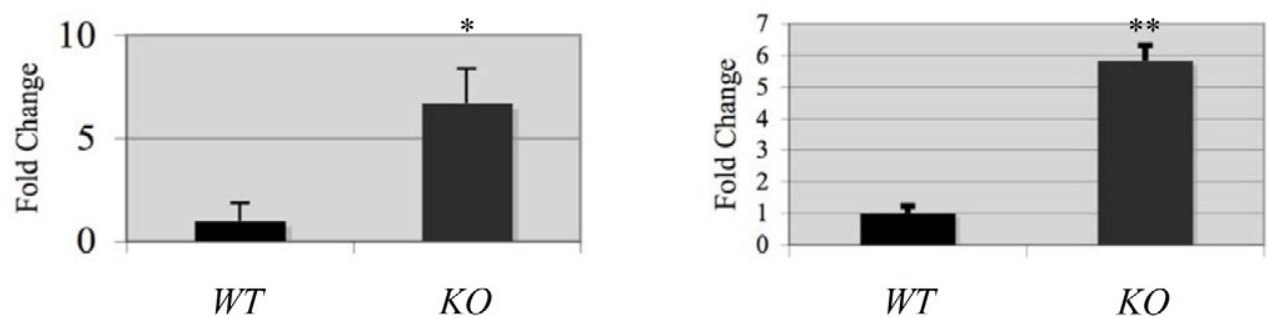

Figure 3. Increased angiogenesis in Smad4 KO wounds. A: H\&E staining reveals the appearance of abnormally large blood vessels in Smad4 KO wounds (black arrows) not seen in wild-type (WT) controls. B: CD31 staining in non-wounded (day 0) and wounded (day 3 ) skin. The dotted lines in the day 3 panels highlight the wound margin. Scale bars in (A) and (B) are $200 \mu \mathrm{m}$ for all panels. C: Quantification of CD31 between wild-type and Smad4 KO skin. *** $P<0.01$. D and E: VEGFA mRNA was increased during both day 0 and day 3 in $\operatorname{Smad} 4 K O$ wounds. Relative VEGFA level of wild-type controls in each group was arbitrarily set as 1 . ${ }^{*} P<0.05$. ${ }^{* * 1 *} P<0.01$.

scars remained in Smad4 KO skin after 6 weeks of healing, with a poorly organized ECM and less accumulation of well-formed collagen fibrils (Figure 5B). Finally, PicroSirius red staining revealed that Smad4 KO wounds exhibited lower collagen content and thinner fibrils compared with wild-type wounds (Figure 5C).

\section{Smad4 KO Skin and Wounds Had Increased TGF $\beta$ and MMP Levels}

The similarities of the wound healing defects in Smad4 $K O$ mice and TGF $\beta 1$ transgenic mice ${ }^{6}$ led us to assess 


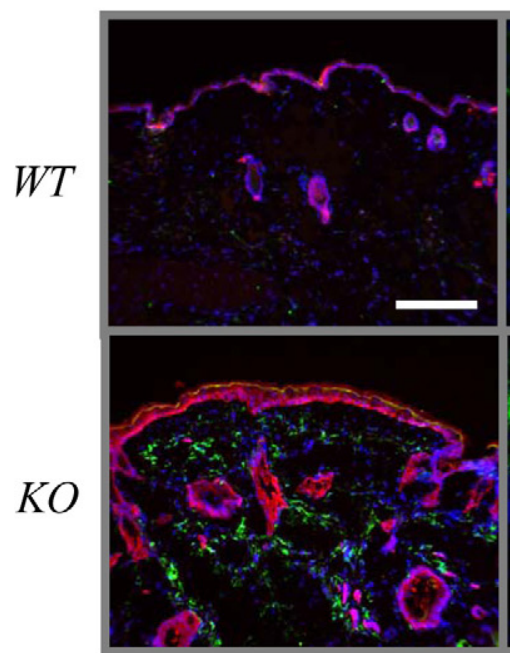

Day 0

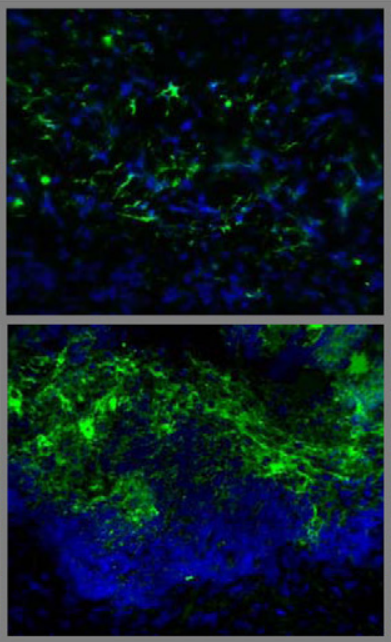

Day 5

\section{B Fold Change in CCL5 mRNA}

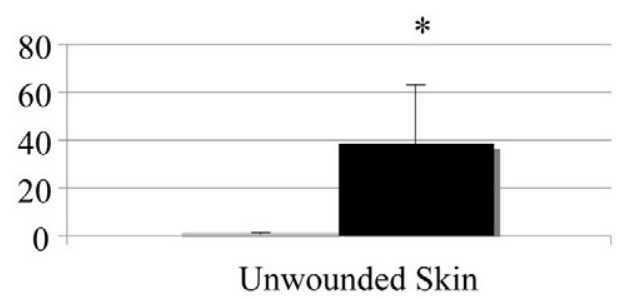

D Fold Change in CCL5 mRNA

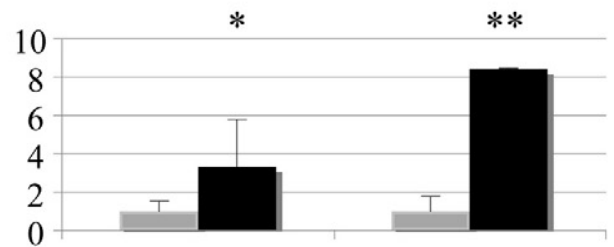

Day 3

Day 5

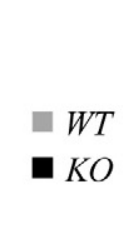

$\mathrm{KO}$

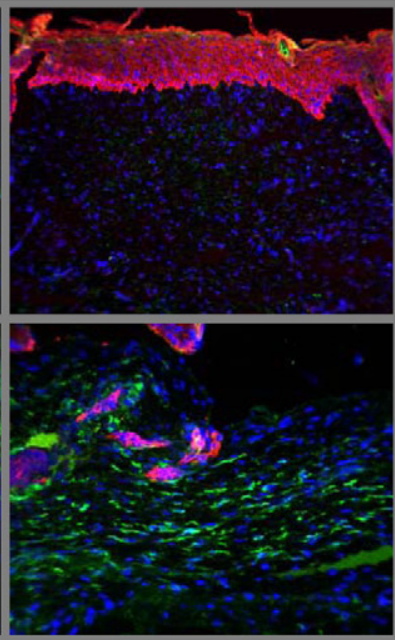

Day 14

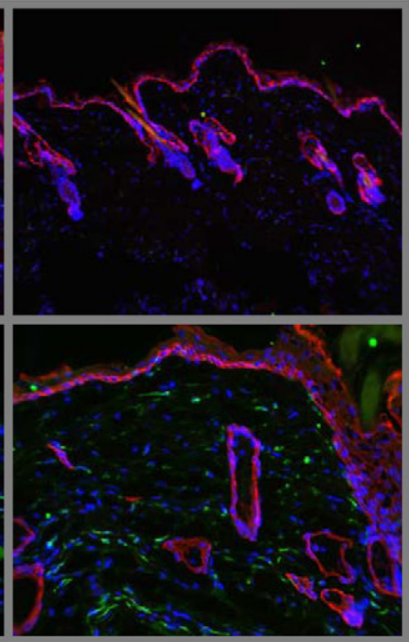

Day 42

\section{Fold Change in CCL20 mRNA}

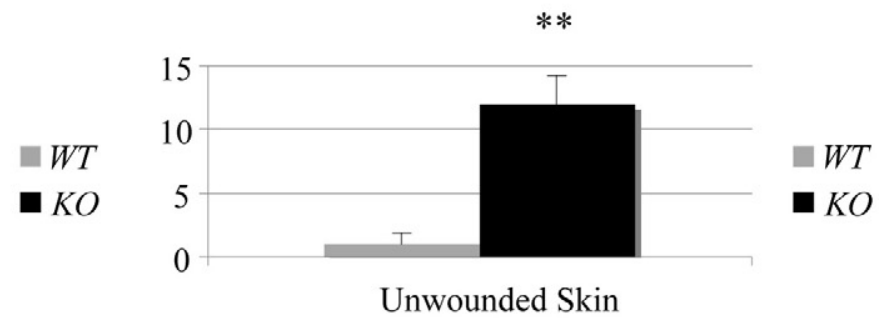

\section{E Fold Change in CCL20 mRNA}

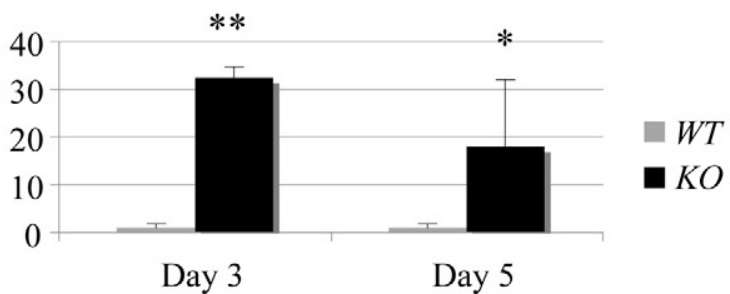

Figure 4. Smad4 KO skin and wounds had increased monocyte/macrophage infiltrates. A: Immunofluorescence staining depicts the monocyte/macrophage marker F4/80 (green) with keratinocytes labeled with K14 (red) and nuclei by 4,6-diamidino-2-phenylindole (blue). The scale bar $=200 \mu \mathrm{m}$ for all panels in $(\mathbf{A})$. B-E: Real-time PCR of mRNA from unwounded skin, day 3 and day 5 wounds for log-fold change of CCL5 and CCL20 mRNA with four wounds from each group assayed. Relative mRNA level of wild-type (WT) controls in each group was arbitrarily set as $1 .{ }^{*} P<0.05$. ${ }^{* *} P<0.01$.

whether endogenous TGF $\beta 1$ is increased in Smad4 KO skin. Quantitative reverse transcription-PCR showed elevated levels of TGF $\beta 1$ mRNA in Smad4 KO skin in comparison with wild-type skin (Figure 6A). During normal wound healing, TGF $\beta 1$ is increased and reaches its peak level on day 3 , followed by a steady decrease thereafter. ${ }^{6}$ Smad4 KO wounds on day 5 had further elevated levels of TGF $\beta 1$, as compared with wild-type wounds (Figure 6A), and remained greater than wild-type levels 6 weeks postwounding (data not shown). Immunostaining using a TGF $\beta 1$ antibody revealed that TGF $\beta 1$ staining was mainly located in the dermis of wild-type skin, but was increased primarily in keratinocytes of unwounded Smad4 KO skin (Figure 6B). Increased TGF $\beta 1$ staining was detected in both epidermis and stroma of wild-type wounds (Figure 6B). However, Smad4 KO wounds showed stronger TGF $\beta 1$ staining intensity in both the epidermis and stroma beginning on day 3 (not shown) and peaked on day 5 (Figure 6B). To assess if increased TGF $\beta 1$ signals through Smad2 and Smad3 in Smad4 KO skin, we stained tissue sections for phosphorylated Smad2 (pSmad2), a surrogate marker for activation of Smad-dependent TGF $\beta$ signaling. Increased pSmad2 nuclear staining was detected in both unwounded epidermis and hair follicles of Smad4 KO skin when compared with wildtype skin (Figure 6B). In wounded skin, increased pSmad2 nuclear staining was detected in both the migrating epidermis and stroma of Smad4 KO skin when compared 
A

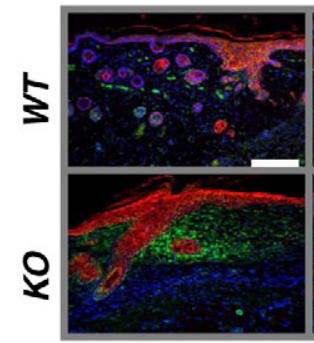

Day 3
aSMA/K14/DAPI

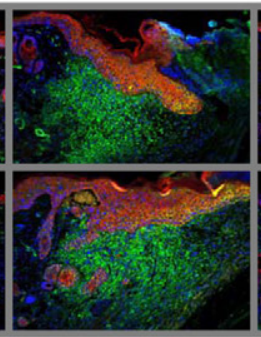

Day 5

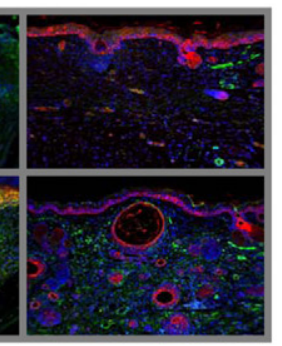

Day 14

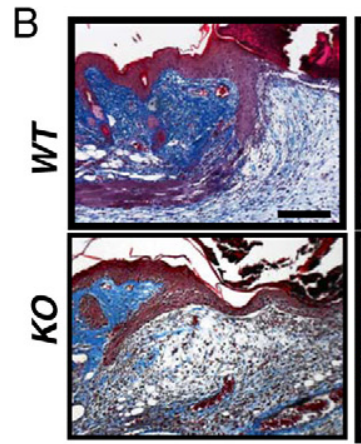

Day 3

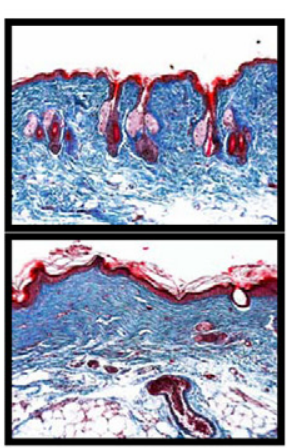

Day 42
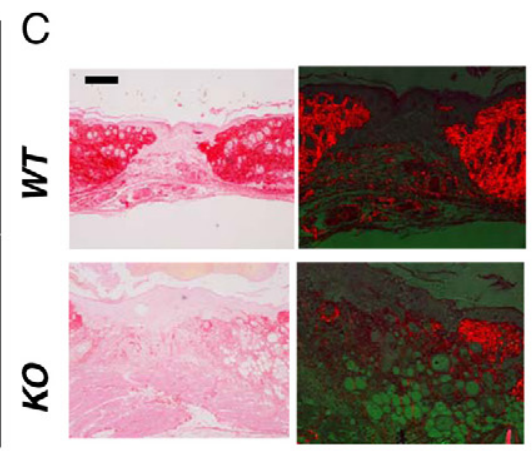

Day 3
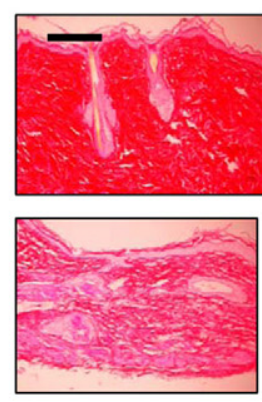

Day 42
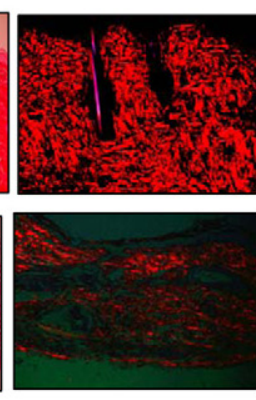

(Figure 3), expression levels of these molecules were further increased in Smad4 KO SCC samples in comparison with Smad4 KO wounds (Figure 8, A-C, E). Additionally, Smad4 KO SCCs exhibited expression levels of MMP3, -12, and -13 similar to those in Smad4 KO skin (not shown), whereas MMP14 showed further elevation in Smad4 KO SCCs, in comparison with Smad4 KO skin (Figure 8D). during wound healing, in comparison with wild-type unwounded and wounded skin at the same stages.

\section{Common Molecular and Cellular Alterations between Wounded Skin and Spontaneous Skin Squamous Cell Carcinomas in Smad4 KO Mice}

Intrigued by a hypothesis that tumors are 'wounds that do not heal,' ${ }^{23}$ we examined whether changes in Smad4 KO wounds were similar to changes observed in spontaneous skin squamous cell carcinomas (SCCs) developed from Smad4 KO skin. First, consistent with the Smad4 KO skin and wounds, Smad4 KO SCCs were highly vascularized (Figure 7A) as shown by staining of CD31 for vessels. $\alpha$ SMA staining demonstrated massive numbers of myofibroblasts in tumor stroma (Figure 7B). Smad4 KO SCCs had abundant monocyte/macrophage infiltrates as shown by staining for F4/80 (Figure 7C). Additionally, Masson trichrome staining and PicroSirius red staining revealed degraded ECM in Smad4 KO SCC stroma (Figure 7, D-F). After ascertaining the multiple histological similarities between wounds and tumors, we compared molecular changes in Smad4 KO SCCs to Smad4 KO unwounded skin using quantitative reverse transcriptionPCR. Similar to the increased expression of VEGFA, CCL5, CCL20, and TGF $\beta 1$ in Smad4 KO wounded skin

\section{Discussion}

\section{Epidermal Smad4 Loss Affects the Stroma in Wound Healing and Tumorigenesis}

Smad4 has been widely described as a tumor suppressor. ${ }^{2,24-28}$ Recently, experiments in cultured cells have shown that a knockdown of Smad4 results in a cell migration defect. ${ }^{12,18}$ We have also observed that primary keratinocytes exhibit this defect in tissue culture (Figure 2). These in vitro observations conflict with the fact that Smad4 deletion results in more invasive tumors. Our data show that in vivo, the hyperproliferative effect and the stromal changes as a result of epithelial Smad4 loss, can overcome the intrinsic migration defect of Smad4 KO cells shown in tissue culture. In contrast, wound healing defects in Smad4 KO skin appeared to result from excessive angiogenesis/inflammation and not from the epithelium where Smad4 is disrupted.

We have shown TGF $\beta 1$ overexpression is common in human skin cancers, ${ }^{29}$ and TGF $\beta 1$ overexpression in keratinocytes results in inflammation, angiogenesis and fibrosis. ${ }^{7}$ In this study, we found that TGF $\beta 1$ was elevated in Smad4 KO skin before wounding or SCC formation, 
A

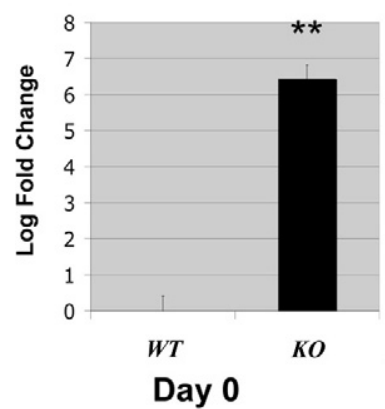

B
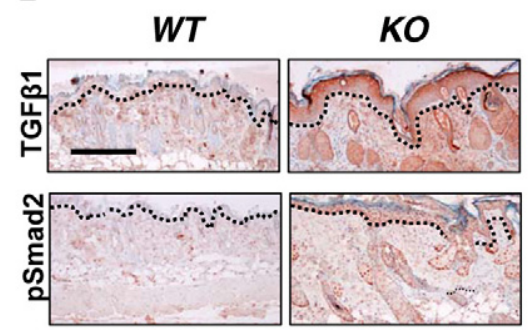

Unwounded
Relative TGF $\beta 1$ mRNA levels

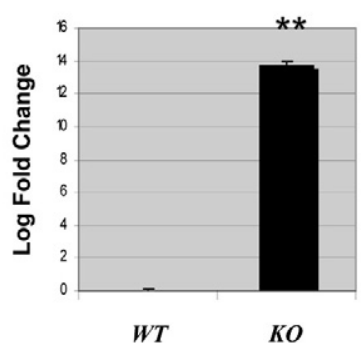

Day 3

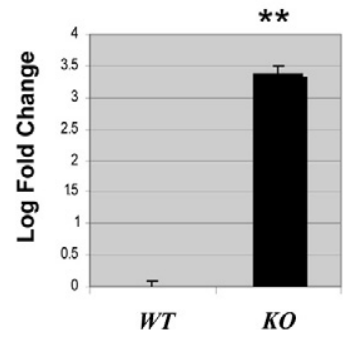

Day 5

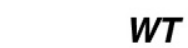

WT
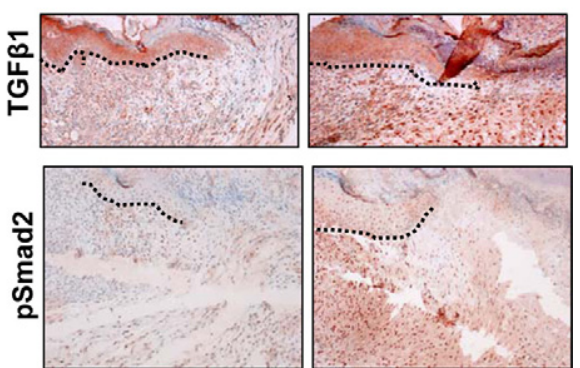

Day 5

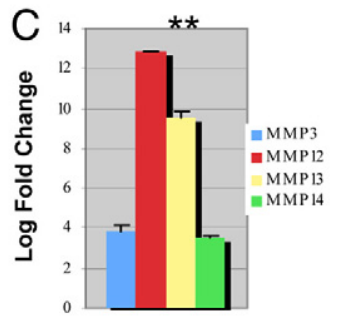

Day 0

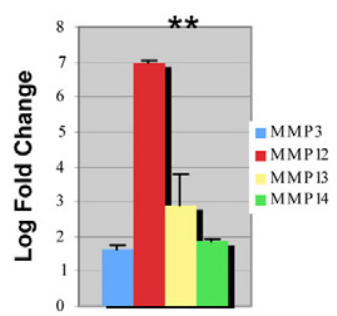

Day 3

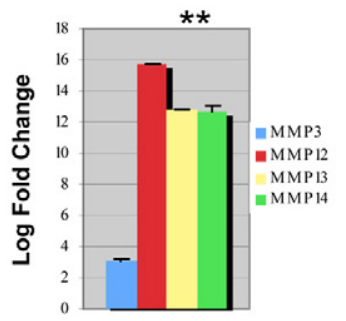

Day 5
Figure 6. TGF $\beta 1$ was increased in Smad4 $K O$ skin and wound. A: Real-time PCR for TGF $\beta 1$ mRNA demonstrates up-regulation of TGF $\beta 1$ in Smad4 KO skin before wounding and during the height of the re-epithelialization phase of wound healing, as compared with healed skin at the same time points. Relative mRNA level of wildtype (WT) controls in each group was arbitrarily set as 1 , ie, 0 in $\log$ scale. ${ }^{* *} P<0.01$. B: Immunohistochemical staining of TGF $\beta 1$ and $\mathrm{pSmad} 2$ in non-wounded and wounded (Day 5) skin. Increased TGF $\beta 1$ staining and pSmad2 nuclear staining were found mainly in keratinocytes of unwounded Smad4 KO skin, and in both epidermis and stroma in Smad4 KO wounds in comparison with wild-type controls. Dotted lines highlight epidermal-stromal boundaries The scale bar $=200 \mu \mathrm{m}$ for all panels in $(\mathbf{B})$ Similar findings were found at other time points of wounded skin (not shown). C: Real-time PCR for MMPs is shown as a relative fold from wildtype skin or wounds. All groups contained at least four samples. Relative mRNA level of wildtype controls in each group was arbitrarily set as 1 , ie, 0 in $\log$ scale. ${ }^{* * *} P<0.01$ for all MMPs. and was further increased under these two pathological conditions. While TGF $\beta 1$ elevation might allow for endogenous tumor suppressive roles, the lack of Smad4 overrides these benefits because the cells no longer signal to induce growth arrest. However, TGF $\beta 1$-induced stromal effects appeared to be independent of epithelial Smad4 signaling. Increased TGF $\beta 1$ may signal through Smadindependent pathways, ${ }^{4}$ which could contribute to pathological alterations in Smad4 KO tissues. Additionally, because TGF $\beta 1$ is a secreted protein, it is possible that nonepithelial cells, which still have intact Smad4, conferred increased TGF $\beta$ signaling required for inflammation, angiogenesis and fibrotic response. Lastly, the remaining Smad2 and Smad3 in keratinocytes may transduce TGF $\beta$ signaling in the absence of Smad4. Supporting the last two possibilities, both epidermal and stromal cells showed increased nuclear pSmad2 in Smad4 KO skin (Figure 6). Moreover, several potential TGF $\beta$ target genes with protumorigenic functions, including CCL5, ${ }^{19,21,30}$ CCL20, $22,31-34$ VEGFA, and MMPs, were elevated in Smad4 KO skin and during wound healing. Some of these molecules (eg, CCL5, CCL20, VEGFA, and MMP14) were further elevated in Smad4 KO SCCs when compared with Smad4 KO wounds, which could be caused by further elevation of TGF $\beta 1$ levels or additional oncogenic alterations in Smad4 KO SCCs. Kinzler and Vogelstein ${ }^{35}$ first described the loss of Smad4 to elicit 'landscape' changes in the tumor microenvironment. Consistent with this notion, we found that, in the wound environment, the real significance of the Smad4 epithelial loss is its indirect effects on the stroma. Smad4 spontaneous tumors tend to develop between 3 to 13 months after birth. ${ }^{2}$ While this observation suggests additional genetic alterations are required for carcinoma formation, Smad4 loss-associated stromal effects may play a role in promoting malignant transformation.

\section{Increased Angiogenesis in Smad4 KO Skin Contributes to Delayed Wound Healing and Remodeling}

We observed enlarged vessels and increased angiogenesis in Smad4 KO skin before wounding. This phenotype may be explained by several observations. First, we previously observed collapsed hair follicles and a hyperproliferative epidermis in Smad4-deficient skin. ${ }^{2,36}$ The hair follicle cysts and hyperplastic epidermis may require a 


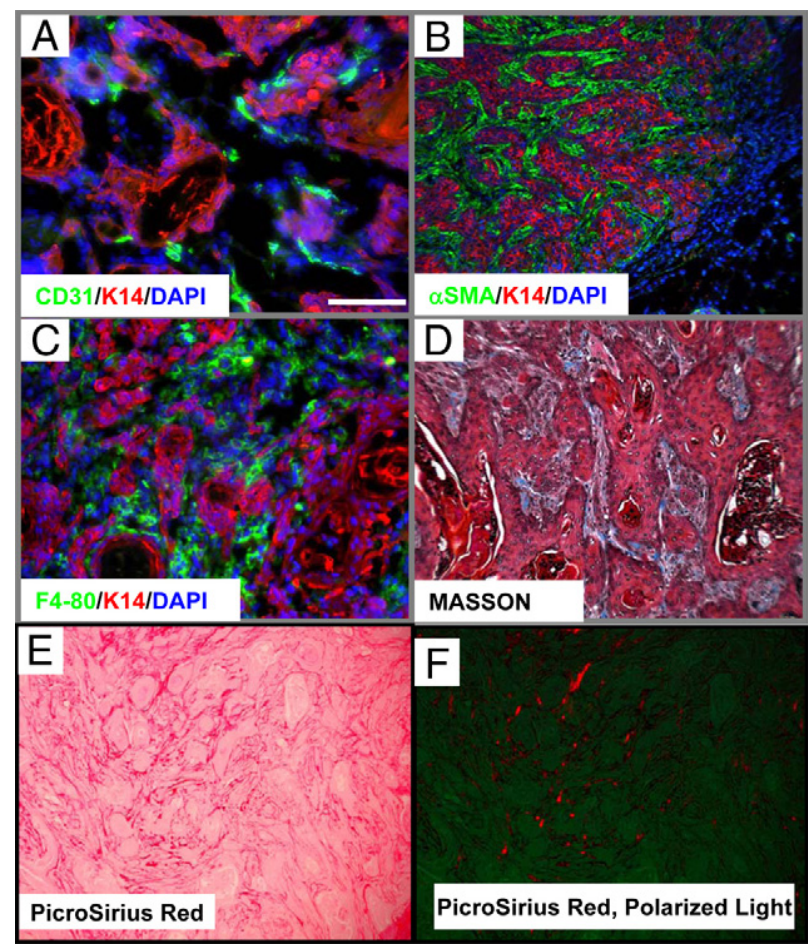

Figure 7. Smad4 KO spontaneous tumors exhibited pathological changes similar to Smad4 KO wounds. A-C: K14 (red) labels keratinocytes and 4,6-diamidino-2-phenylindole (blue) shows nuclei (A) CD31 (green) immunofluorescence staining showed that $\operatorname{Smad} 4 \mathrm{KO}$ spontaneous tumors were highly vascularized. B: $\alpha$ SMA (green) immunofluorescence staining shows myofibroblasts accumulating in the surrounding stroma. C: F4/80 (green) immunofluorescence staining shows massive monocyte/macrophage infiltration in the tumor stroma. D: Masson trichrome staining of Smad4 KO tumors demonstrates the nature of the degraded ECM by lack of blue staining surrounding the tumor. $\mathbf{E}$ and $\mathbf{F}$ : Reduced collagen in SCC stroma revealed by PicroSirius red staining viewed through a bright field (E) and polarized light (F). The green background under the polarized light highlights the entire SCC epithelia and stroma. The scale bar in the first panel represents $200 \mu \mathrm{m}$ for all panels.

substantial blood supply to fuel their aberrant growth. Second, the levels of TGF $\beta 1$, a potent stimulator of new blood vessel formation, were increased in Smad4 KO skin. During wound healing, angiogenesis is necessary to reestablish blood flow to newly formed epithelia. However, excessive angiogenesis further facilitates leukocyte infiltration and provides circulating cytokines/chemokines, resulting in unresolved inflammation. This in turn, could cause a delay in ECM remodeling and wound contraction. Our data indicate that a wound in the preexisting angiogenic environment coupled with unresolved angiogenesis caused by continuous overexpression of TGF $\beta 1$ and VEGFA during wound healing leads to delayed healing in Smad4 KO wounds.

\section{Excessive Inflammation in Smad4 KO Skin Contributes to Delayed Wound Remodeling}

Inflammation brings macrophages to the wound, which are necessary for phagocytosis of debris from the wound area. However, if these inflammatory cells remain in the wound for an extended period, normal remodeling of the stroma cannot occur. The severity of macrophage infiltration in non-wounded and wounded skins of Smad4 KO mice correlated with their expression levels of TGF $\beta 1$, one of the most potent chemotactic cytokines for macrophage infiltration. ${ }^{37,38}$ Therefore, TGF $\beta 1$ overexpression could be a major contributing factor for persistent macrophage infiltration in Smad4 KO tissues. Exacerbation of the macrophage response impairs wound healing when it competes with repopulation of the dermis by ECM molecules, resulting in an inhibition of proper matrix remodeling and an excessive scar. Hence both pre-existing inflammation and exacerbated inflammation during wound healing could contribute to the improper healing of Smad4 $K O$ wounds. Persistent macrophage infiltration may play a role in the malignant progression of Smad4 KO tumors, as tumor associated macrophages are known to promote carcinoma formation. ${ }^{38}$ Our current data are consistent with the previous report that an elevation of TGF $\beta 1$ in skin tumors results in the recruitment of tumor associated macrophages. ${ }^{38}$
A
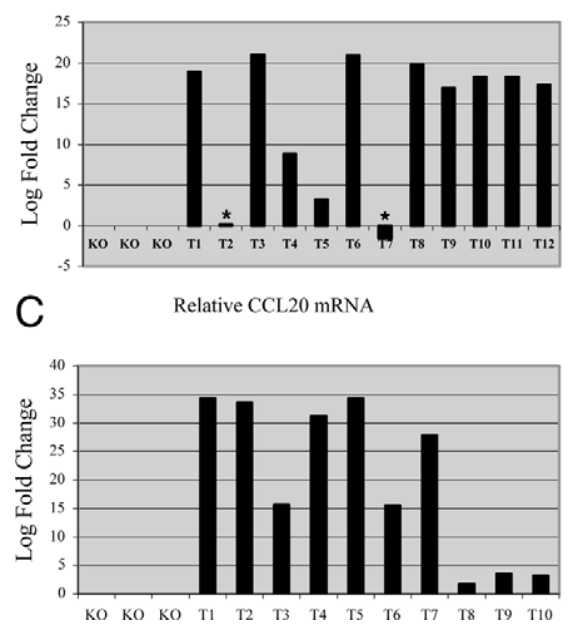

B

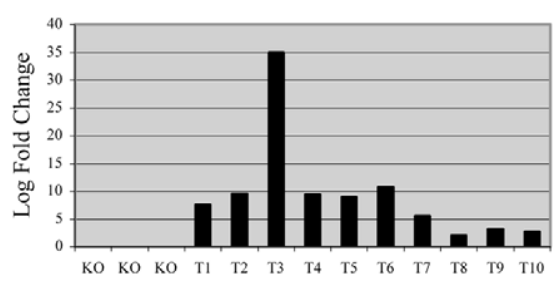

D

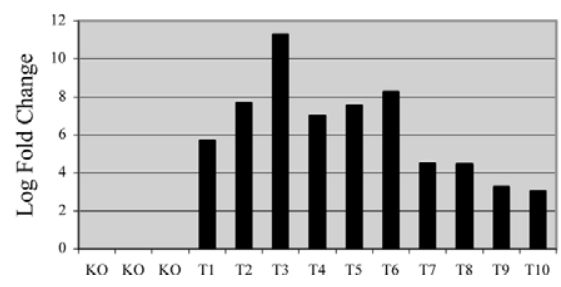

Figure 8. Smad4 KO spontaneous tumors exhibited molecular changes similar to Smad4 KO wounds. qRT- PCR compares at least three Smad4 $K O$ skins with at least ten tumors for mRNA of VEGFA (A), CCL5 (B), CCL20 (C), MMP14 (D), and $\operatorname{TGF} \beta(\mathbf{E})$ are all significantly increased $(P<$ $0.01)$. Relative mRNA level of wild-type (WT) controls in each group was arbitrarily set as 1, ie, 0 in $\log$ scale. Asterisks in A: no further increase compared with Smad4 KO skin.

\section{E Relative TGF $\beta 1$ mRNA}

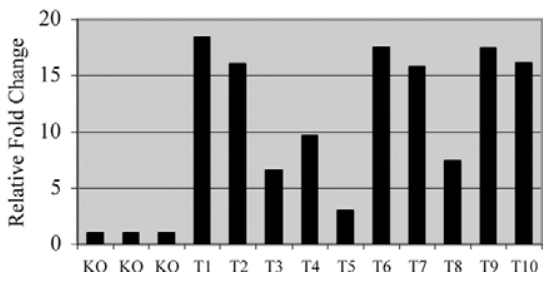




\section{Persistent Myofibroblast Accumulation in Smad4 KO Skin Contributes to Delayed Wound Remodeling}

Myofibroblasts are required in normal wound healing for wound contraction and ECM formation. However, the persistence of myofibroblasts beyond a normal time frame in wound healing can lead to fibrosis and delayed wound remodeling. TGF $\beta 1$ has been shown to promote fibroblast differentiation to the myofibroblast state ${ }^{39}$ and these myofibroblast cells have been shown to facilitate tumor progression. ${ }^{40}$ The correlation between increased proliferative stromal cells and TGF $\beta 1$ overexpression in Smad4 KO skin and wounds suggests that persistent TGF $\beta 1$ overexpression and inflammation may be major contributing factors to increased myofibroblast numbers in Smad4 KO wounds and tumors. Further, although TGF $\beta 1$ is a potent growth inhibitor for epithelial cells, it also stimulates fibroblast proliferation and myofibroblast activation. ${ }^{6}$ These facts indicate that the Smad4 deficiency in the epithelium is sufficient to result in the stromal accumulation of myofibroblasts to pathological levels. The accumulated myofibroblasts in Smad4 KO wounds could prevent normal ECM molecule deposition during wound remodeling, causing delayed scar resolution.

In summary, we report that epidermal Smad4 deletion has a significant impact on the stroma, which causes delayed wound closure and remodeling. The excessive inflammation, angiogenesis, and myofibroblasts in Smad $4 \mathrm{KO}$ wounds were further exacerbated in Smad4 KO skin tumors. Future studies on the later stages of wound healing, particularly the mechanisms of receding angiogenesis, and reducing inflammation and myofibroblasts will further our understanding of the mechanisms of Smad 4 and TGF $\beta$ signaling in wound healing and skin cancer and will ultimately help us to effectively heal wounds and treat cancer.

\section{Acknowledgments}

We thank Dr. Chuxia Deng for providing Smad4 floxed mice, Carolyn Gendron for histology advice, and Miss Pamela Garl for critical proof reading of the manuscript.

\section{References}

1. Singer AJ, Clark RA: Cutaneous wound healing. N Engl J Med 1999, 341:738-746

2. Qiao W, Li AG, Owens P, Xu X, Wang XJ, Deng CX: Hair follicle defects and squamous cell carcinoma formation in Smad4 conditional knockout mouse skin. Oncogene 2006, 25:207-217

3. Hubner $\mathrm{G}, \mathrm{Hu} \mathrm{Q}$, Smola $\mathrm{H}$, Werner S: Strong induction of activin expression after injury suggests an important role of activin in wound repair. Dev Biol 1996, 173:490-498

4. Derynck R, Zhang Y: Smad-dependent and Smad-independent pathways in TGF-beta family signalling. Nature 2003, 425:577-584

5. Wang Z, Gao Z, Shi Y, Sun Y, Lin Z, Jiang H, Hou T, Wang Q, Yuan $X$, Zhu $X$, Wu H, Jin Y: Inhibition of Smad3 expression decreases collagen synthesis in keloid disease fibroblasts. J Plast Reconstr Aesthet Surg 2007, 60:1193-1199

6. Wang XJ, Han G, Owens P, Siddiqui Y, Li AG: Role of TGF betamediated inflammation in cutaneous wound healing. J Investig Dermatol Symp Proc 2006, 11:112-117
7. Li AG WD, Feng XH, Wang XJ: Latent TGFbeta1 overexpression in keratinocytes results in a severe psoriasis-like skin disorder. EMBO 2004, 23:1770-1781

8. Yang L, Chan T, Demare J, Iwashina T, Ghahary A, Scott PG, Tredget EE: Healing of burn wounds in transgenic mice overexpressing transforming growth factor-beta 1 in the epidermis. Am J Pathol 2001, 159:2147-2157

9. Tredget EB, Demare J, Chandran G, Tredget EE, Yang L, Ghahary A: Transforming growth factor-beta and its effect on reepithelialization of partial-thickness ear wounds in transgenic mice. Wound Repair Regen 2005, 13:61-67

10. Ashcroft GS, Yang X, Glick AB, Weinstein M, Letterio JL, Mizel DE, Anzano M, Greenwell-Wild T, Wahl SM, Deng C, Roberts AB: Mice lacking Smad3 show accelerated wound healing and an impaired local inflammatory response. Nat Cell Biol 1999, 1:260-266

11. Hosokawa R, Urata MM, Ito $Y$, Bringas $P$, Jr., Chai $Y$ : Functional significance of Smad2 in regulating basal keratinocyte migration during wound healing. J Invest Dermatol 2005, 125:1302-1309

12. Levy L, Hill CS: Smad4 dependency defines two classes of transforming growth factor beta (TGF-beta) target genes and distinguishes TGF-beta-induced epithelial-mesenchymal transition from its antiproliferative and migratory responses. Mol Cell Biol 2005, 25:8108-8125

13. Hoot KE, Lighthall J, Han G, Lu SL, Li A, Ju W, Kulesz-Martin M, Bottinger E, Wang $\mathrm{XJ}$ : Keratinocyte-specific Smad2 ablation results in increased epithelial-mesenchymal transition during skin cancer formation and progression. J Clin Invest 2008, 118:2722-2732

14. Yang X, Li C, Herrera PL, Deng CX: Generation of Smad4/Dpc4 conditional knockout mice. Genesis 2002, 32:80-81

15. Wagner KU, Wall RJ, St-Onge L, Gruss P, Wynshaw-Boris A, Garrett L, Li M, Furth PA, Hennighausen L: Cre-mediated gene deletion in the mammary gland. Nucleic Acids Res 1997, 25:4323-4330

16. Zambrowicz BP, Imamoto A, Fiering S, Herzenberg LA, Kerr WG, Soriano P: Disruption of overlapping transcripts in the ROSA beta geo 26 gene trap strain leads to widespread expression of beta-galactosidase in mouse embryos and hematopoietic cells. Proc Natl Acad Sci USA 1997, 94:3789-3794

17. Cuttle L, Nataatmadja M, Fraser JF, Kempf M, Kimble RM, Hayes MT: Collagen in the scarless fetal skin wound: detection with picrosiriuspolarization. Wound Repair Regen 2005, 13:198-204

18. Jazag A, Ijichi H, Kanai F, Imamura T, Guleng B, Ohta M, Imamura J, Tanaka Y, Tateishi K, Ikenoue T, Kawakami T, Arakawa Y, Miyagishi M, Taira K, Kawabe T, Omata M: Smad4 silencing in pancreatic cancer cell lines using stable RNA interference and gene expression profiles induced by transforming growth factor-beta. Oncogene 2005, 24:662-671

19. Karnoub AE, Dash AB, Vo AP, Sullivan A, Brooks MW, Bell GW, Richardson AL, Polyak K, Tubo R, Weinberg RA: Mesenchymal stem cells within tumour stroma promote breast cancer metastasis. Nature 2007, 449:557-563

20. Chang KP, Hao SP, Chang JH, Wu CC, Tsang NM, Lee YS, Hsu CL, Ueng SH, Liu SC, Liu YL, Wei PC, Liang Y, Chang YS, Yu JS: Macrophage inflammatory protein-3alpha is a novel serum marker for nasopharyngeal carcinoma detection and prediction of treatment outcomes. Clin Cancer Res 2008, 14:6979-6987

21. Borczuk AC, Papanikolaou N, Toonkel RL, Sole M, Gorenstein LA Ginsburg ME, Sonett JR, Friedman RA, Powell CA: Lung adenocarcinoma invasion in TGFbetaRII-deficient cells is mediated by CCL5/ RANTES. Oncogene 2008, 27:557-564

22. Giuliani N, Lisignoli G, Colla S, Lazzaretti M, Storti P, Mancini C, Bonomini S, Manferdini C, Codeluppi K, Facchini A, Rizzoli V: CC-Chemokine ligand 20/macrophage inflammatory protein-3alpha and CC-chemokine receptor 6 are overexpressed in myeloma microenvironment related to osteolytic bone lesions. Cancer Res 2008, 68:6840-6850

23. Dvorak HF: Tumors: wounds that do not heal. Similarities between tumor stroma generation and wound healing. N Engl J Med 1986, 315:1650-1659

24. Bardeesy N, Cheng KH, Berger JH, Chu GC, Pahler J, Olson P, Hezel AF, Horner J, Lauwers GY, Hanahan D, DePinho RA: Smad4 is dispensable for normal pancreas development yet critical in progression and tumor biology of pancreas cancer. Genes Dev 2006, 20:3130-3146

25. Xiao DS, Wen JF, Li JH, Wang KS, Hu ZL, Zhou JH, Deng ZH, Liu Y: Effect of DPC4 gene on invasion and metastasis of colorectal carcinoma cells. Acta Biochim Biophys Sin (Shanghai) 2006, 38:883-892 26. Yang L, Mao C, Teng Y, Li W, Zhang J, Cheng X, Li X, Han X, Xia Z, 
Deng H, Yang X: Targeted disruption of Smad4 in mouse epidermis results in failure of hair follicle cycling and formation of skin tumors. Cancer Res 2005, 65:8671-8678

27. Kim BG, Li C, Qiao W, Mamura M, Kasprzak B, Anver M, Wolfraim L, Hong S, Mushinski E, Potter M, Kim SJ, Fu XY, Deng C, Letterio JJ: Smad4 signalling in $T$ cells is required for suppression of gastrointestinal cancer. Nature 2006, 441:1015-1019

28. Kitamura T, Kometani K, Hashida H, Matsunaga A, Miyoshi H, Hosogi $\mathrm{H}$, Aoki M, Oshima M, Hattori M, Takabayashi A, Minato N, Taketo MM: SMAD4-deficient intestinal tumors recruit CCR1+ myeloid cells that promote invasion. Nat Genet 2007, 39:467-475

29. Han G, Lu SL, Li AG, He W, Corless CL, Kulesz-Martin M, Wang XJ: Distinct mechanisms of TGF-beta1-mediated epithelial-to-mesenchymal transition and metastasis during skin carcinogenesis. J Clin Invest 2005, 115:1714-1723

30. Soria G, Ben-Baruch A: The inflammatory chemokines CCL2 and CCL5 in breast cancer. Cancer Lett 2008, 267:271-285

31. Li HX, Chen P, Wang L, Xu JR, Wang GQ, Jia YQ, Yang L: [Effect of lenti-mCCL20 on the growth of mouse tumor]. Sichuan Da Xue Xue Bao Yi Xue Ban 2006, 37:712-716

32. Kimsey TF, Campbell AS, Albo D, Wilson M, Wang TN: Co-localization of macrophage inflammatory protein-3alpha (Mip-3alpha) and its receptor, CCR6, promotes pancreatic cancer cell invasion. Cancer J 2004, 10:374-380
33. Campbell AS, Albo D, Kimsey TF, White SL, Wang TN: Macrophage inflammatory protein-3alpha promotes pancreatic cancer cell invasion. J Surg Res 2005, 123:96-101

34. Abiko Y, Nishimura M, Kusano K, Nakashima K, Okumura K, Arakawa T, Takuma T, Mizoguchi I, Kaku T: Expression of MIP-3alpha/CCL20 a macrophage inflammatory protein in oral squamous cell carcinoma. Arch Oral Biol 2003, 48:171-175

35. Kinzler KW, Vogelstein B: Landscaping the cancer terrain. Science 1998, 280:1036-1037

36. Owens $P$, Bazzi H, Engelking E, Han G, Christiano AM, Wang XJ: Smad4-dependent desmoglein-4 expression contributes to hair follicle integrity. Dev Biol 2008, 322:156-166

37. McCartney-Francis NL, Wahl SM: Transforming growth factor beta: a matter of life and death. J Leukoc Biol 1994, 55:401-409

38. Byrne SN, Knox MC, Halliday GM: TGFbeta is responsible for skin tumour infiltration by macrophages enabling the tumours to escape immune destruction. Immunol Cell Biol 2008, 86:92-97

39. Narine K, DeWever O, Cathenis K, Mareel M, Van Belleghem Y, Van Nooten G: Transforming growth factor-beta-induced transition of fibroblasts: a model for myofibroblast procurement in tissue valve engineering. J Heart Valve Dis 2004, 13:281-289; discussion 289

40. De Wever O, Demetter P, Mareel M, Bracke M: Stromal myofibroblasts are drivers of invasive cancer growth. Int J Cancer 2008 123:2229-2238 